Wesley de Castro Fagundes

\title{
Estimativa do Fator de Carga (FC) e Fator de Demanda (FD) de Consumidores de Energia Elétrica Via Medição e Pesquisa de Posses e Hábitos
}

Dissertação apresentada como requisito parcial para obtenção do título de Mestre pelo Programa de PósGraduação em Metrologia (Área de concentração: Metrologia para Qualidade e Inovação) da PUC-Rio.

Orientador: Prof. Reinaldo Castro Souza Co-orientador: Prof. Maurício Nogueira Frota 
Wesley de Castro Fagundes

\section{Estimativa do Fator de Carga (FC) e Fator de Demanda (FD) de Consumidores de Energia Elétrica Via Medição e Pesquisa de Posses e Hábitos}

Dissertação apresentada como requisito parcial para obtenção do título de Mestre pelo Programa de PósGraduação em Metrologia (Área de concentração: Metrologia para Qualidade e Inovação) da PUC-Rio. Aprovada pela Comissão Examinadora abaixo assinada.

Comissão Examinadora:

Prof. Reinaldo Castro Souza

Orientador

Programa de Pós-Graduação em Metrologia (PósMQI/PUC-Rio) Pontifícia Universidade Católica do Rio de Janeiro

Prof. Maurício Nogueira Frota

Co-orientador

Programa de Pós-Graduação em Metrologia (PósMQI/PUC-Rio) Pontifícia Universidade Católica do Rio de Janeiro

Prof. André Luís Marques Marcato Universidade Federal de Juiz de Fora

Prof. João Carlos de Oliveira Aires

Universidade Gama Filho

Coordenação Setorial de Pós-Graduação:

Prof. José Eugenio Leal Coordenador Setorial de Pós-Graduação do Centro Técnico Científico (PUC-Rio) 
Todos os direitos reservados. É proibida a reprodução total ou parcial do trabalho sem autorização da universidade, do autor e do orientador.

\section{Wesley de Castro Fagundes}

Graduou-se em Engenharia Elétrica (2003) pela Universidade Veiga de Almeida. Atualmente possui vínculo com o Instituto de Energia da PUC-Rio, onde trabalha como Assistente de Pesquisa em projetos de P\&D para o setor elétrico.

Ficha Catalográfica

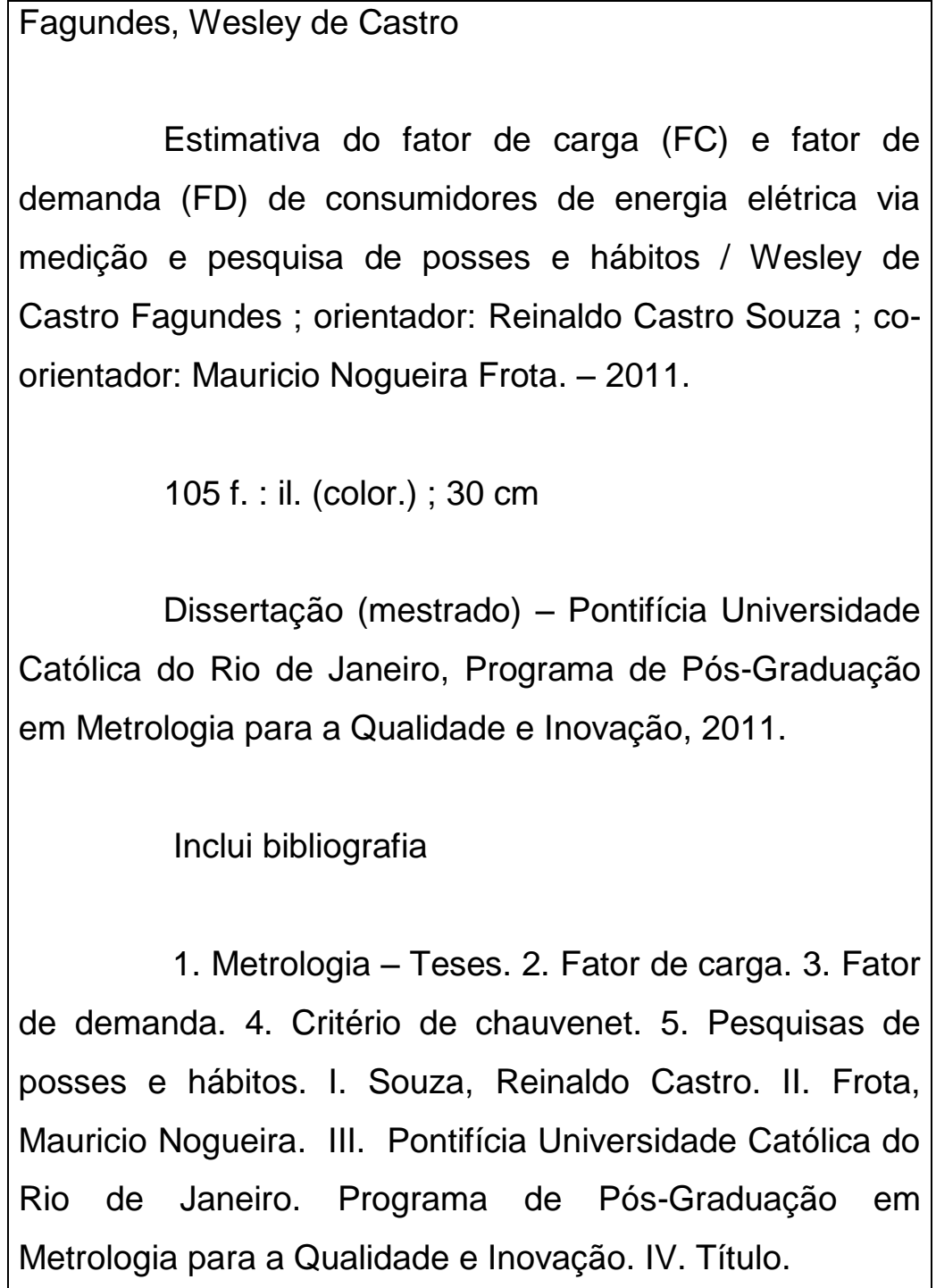


À minha esposa Patrícia e nosso filho João, minhas maiores fontes de inspiração. Agradeço a vocês por toda paciência, amor e compreensão durante este período 


\section{Agradecimentos}

Ao meu orientador, Professor Reinaldo Castro Souza, pela paciência e suporte acadêmico necessário para realização deste trabalho.

Ao meu co-orientador, Professor Mauricio Frota, pela oportunidade de fazer parte do grupo de mestres formados em Metrologia pela PUC.

A minha avó Geraldina, pela lição de vida, motivo de exemplo para todos nós.

A minha mãe, Maria de Lourdes, e meu pai, Victorino, por todo amor e orientações que me foram passadas.

A minha sogra Regina, por todo amor, carinho e dedicação dado a nossa família, em especial ao nosso pequeno João.

A toda minha família, pelo apoio e compreensão nos momentos em que precisei ficar ausente para conclusão deste trabalho.

Ao amigo Rubinho, que muito contribuiu para o levantamento das informações colhidas na pesquisa campo.

Às amigas Elizabeth e Nilmara, pela ajuda nas pesquisas e nas revisões desta tese.

Ao meu grande amigo Vanderlei, que muito incentivou na conclusão desse trabalho.

Para a maravilhosa equipe de funcionários da secretaria de Metrologia, em especial a Márcia Ribeiro, Márcia Bicalho e Paula por estarem sempre dispostas a ajudar.

À Elektro, em especial a Márcia Inoue e Rodrigo Manfredini, pelo empenho e disponibilidade na troca de informações

À PUC-Rio, pelos auxílios concedidos, sem os quais este trabalho não poderia ter sido realizado. 


\section{Resumo}

Fagundes, Wesley de Castro; Souza, Reinaldo Castro. Estimativa do Fator de Carga (FC) e Fator de Demanda (FD) de Consumidores de Energia Elétrica Via Medição e Pesquisa de Posses e Hábitos. Rio de Janeiro, 2011. 105p. Dissertação de Mestrado - Programa de Pós-Graduação em Metrologia. Pontifícia Universidade Católica do Rio de Janeiro.

O objetivo desta dissertação é apresentar os conceitos de Fator de Carga (FC) e Fator de Demanda (FD) e verificar o quanto estas informações estão atualizadas para uso pelas concessionárias de energia elétrica. A motivação pelo estudo resultou do fato de o conhecimento e o gerenciamento desses índices proporciorem maior eficiência e segurança às instalações e equipamentos, além de permitir redução do custo da energia elétrica. A metodologia utilizada para o cálculo se baseia nos testes de Chauvenet, que permite determinar se um valor amostral (resultante de uma medição) é anômalo (outlier) em relação aos restantes valores da amostra, supondo-se que esta amostra é retirada de uma distribuição normal. Dentre os principais resultados destacam-se estimativas do Fator de Carga e Fator de Demanda, típicos para os diversos segmentos de clientes de uma empresa de distribuição de energia elétrica. Para se obter essas estimativas foi realizada uma pesquisa de campo permitindo o levantamento da potência instalada em clientes atendidos na alta e na baixa tensão. Em paralelo à pesquisa de campo, a concessionária de energia elétrica realizou medições de consumo e demanda junto a esses clientes. Como conclusão o trabalho confirma que as distribuidoras de energia elétrica não possuem acesso a um cadastro atualizado da potência instalada de seus consumidores. Faz-se necessário uma atualização mais dinâmica nas bases de dados disponíveis o que permitiria uma estimativa mais precisa dos fatores de carga e de demanda.

\section{Palavras-chave}

Metrologia; Fator de Carga; Fator de Demanda; Critério de Chauvenet; Pesquisas de Posses e Hábitos. 


\section{Abstract}

Fagundes, Wesley de Castro; Souza, Reinaldo Castro (Advisor). Estimation of the Load Factor (LF) and the Demand Factor (DF) of Electricity Consumers via Measurements and Electrical Appliances Ownership and Usage Surveys. Rio de Janeiro, 2011. 105p. MSc. Dissertation Programa de Pós-Graduação em Metrologia. Pontifícia Universidade Católica do Rio de Janeiro.

The aim of this work discusses basic concepts associated with the load factor (LF) and demand factor (DF) and the adequacy of their use by electricity utilities. The knowledge and management of these electrical parameters is rather important, as they contribute for the improvement of the efficiency and security of the system as a whole while promoting reduction on energy costs. The method used to estimate these quantities is based on the Chauvenet statistical tests capable to detect and remove possible discontinuity on the sample data observed to follow normal distribution. The LD and DF were estimated for various clusters of similar clients of a given distributing utility. Estimations were obtained through a survey involving clients of the selected utility to capture information on the end-users and their nominal power demanded at both, the low and high voltage levels. In parallel to the survey, the utility measured and monitored the energy consumption of the clients participating in the study. As a conclusion, the study revealed that the electricity utilities do not have access to update information (stratified by segments of clients) on the electrical parameters. A dynamic scheme to make available updated information related to load factor and demand factor proved to be extremely useful and is highly recommended.

\section{Keywords}

Metrology; Load Factor; Demand Factor; Chauvenet Criterion; Electrical Appliances Ownership Survey. 


\section{Sumário}

1 Introdução 17

1.1. Definição do problema da pesquisa 17

1.2. Objetivos 18

1.3. Motivação 18

1.4. Metodologia 18

1.5. Estrutura da dissertação 19

2 Setor Elétrico Brasileiro $\quad 21$

2.1. Matriz energética nacional 21

2.2. Breve histórico do setor elétrico brasileiro 23

2.3. Os principais agentes do setor elétrico 25

2.3.1. Agência Nacional de Energia Elétrica (ANEEL) 25

2.3.2. Operador Nacional do Sistema Elétrico (ONS) 25

2.3.3. Câmara de Comercialização de Energia Elétrica (CCEE) 27

2.3.4. Empresa de Pesquisa Energética (EPE) 27

2.4. Funcionamento das distribuidoras de energia 28

2.5. Classificação dos consumidores de energia elétrica 28

2.5.1. Grupo A 28

2.5.2. Grupo B 29

2.6. Classes de consumo 29

2.7. Faturamento dos clientes 29

2.8. Medidores 31

3 Posses e hábitos do uso de aparelhos elétricos 34

3.1. Breve histórico 34

3.2. Tipos de pesquisa $\quad 35$

3.3. Plano amostral da PPH para seleção dos municípios 38

3.3.1. Determinação do "perfil elétrico" utilizando análise de cluster 41

3.3.2. Aplicação dos mapas de Kohonen 41

3.3.3. Distâncias entre perfis 43

3.4. Uso da PPH no contexto 44 
4 Fator de carga e fator de demanda: conceituação

4.1. Fator de carga (FC) 45

4.2. Fator de demanda (FD) 45

4.3. Definição ilustrativa do FC e do FD 45

4.4. Disponibilidade de informações nas distribuidoras 49

4.5. Cálculo do FC e FD para um cliente selecionado 52

4.6. Cálculo do FC e FD típicos para grupo de clientes

do mesmo ramo 54

5 Estudo de Caso 55

5.1. Perfil dos consumidores analisados no grupo A 55

5.2. Aplicação da metodologia para um caso real de um ramo de atividade pertencente ao grupo $\mathrm{A}$

6 Conclusões e recomendações para trabalhos futuros

77

7 Referências bibliográficas

79

ANEXO 1 - Instrumento de coleta de dados para levantamento da potência instalada no grupo $A$

ANEXO 2 - Instrumento de coleta de dados para PPH residencial do grupo $B$

APÊNDICE 1 - Tabela de FC e FD típicos utilizada pela

ELEKTRO por atividade - Baixa tensão

APÊNDICE 2 - Tabela de fatores FC e FD típicos utilizados pela ELEKTRO por classe de consumo - Baixa tensão

APÊNDICE 3 - Tabela de fatores FC e FD típicos utilizados pela ELEKTRO por setor industrial - Baixa tensão

APÊNDICE 4 - Tabela de fatores FC e FD típicos utilizados pela ELEKTRO por atividade - Alta tensão 
APÊNDICE 6 - Tabela com os dados de consumo médio (kWh) dos clientes do grupo $B$

APÊNDICE 7 - Tabela com os dados de consumo médio (kWh) dos clientes do grupo B (padronizada) 


\section{Lista de figuras}

Figura 1 - Matriz energética brasileira em 2009

Figura 2 - Oferta interna de energia elétrica no Brasil por fonte em 2009

Figura 3 - Investimento em geração de energia elétrica no Brasil entre 1990 e 1995

Figura 4 - Mapa da área de concessão antes da reestruturação do setor elétrico 24

Figura 5 - Mapa resumido do sistema interligado nacional - SIN 26

Figura 6 - Percurso da energia elétrica até o consumidor final 30

Figura 7 - Medidor Eletromecânico Monofásico 31

Figura 8 - Medidor Eletrônico 32

Figura 9 - Concessionárias que realizaram PPH entre 2004

e 2006 em parceria com a PUC e PROCEL

Figura 10 - Participação de eletrodomésticos no consumo residencial no Brasil em 2005 Fonte:

Figura 11 - Curva de carga diária média na região sudeste

- Ano base 2005

Figura 12 - Simulador SINPHA 38

Figura 13: Topologia da rede $\quad 42$

Figura 14 - Curva de carga residencial para um dia útil 46

Figura 15 - Mapa da área de concessão da ELEKTRO em $2008 \quad 55$

Figura 16 - Mapa atual da área de concessão da ELEKTRO $2011 \quad 56$ 


\section{Lista de Tabelas}

Tabela 1 - Diferenças entre o sistema de medição eletrônica e eletromecânica

Tabela 2 - Levantamento da potência instalada em um apartamento residencial típico

Tabela 3 - Demanda de energia diurna de um apartamento residencial típico

Tabela 4 - Demanda de energia noturna de um apartamento residencial típico

Tabela 5 - Fator de demanda diurno e noturno para um apartamento residencial típico

Tabela 6 - Informações que a concessionária possui por tipo de cliente

Tabela 7 - Quantidade de clientes por classe principal

Tabela 8 - Potência instalada dos clientes amostrados pertencentes ao ramo de atividade "Fabricação de produtos farmacêuticos e veterinários"

Tabela 9 - Histórico das demandas medidas (em kW) dos clientes amostrados pertencentes ao ramo de atividade "Fabricação de produtos farmacêuticos e veterinários"

Tabela 10 - Cálculo dos desvios em relação à média para os valores do histórico de demandas para o cliente C.1

Tabela 12 - Histórico das demandas medidas (em kW) dos clientes amostrados pertencentes ao ramo de atividade "Fabricação de produtos farmacêuticos e veterinários" sem a informação do mês de maio de 2010

Tabela 13 - Cálculo dos desvios em relação à média para os valores do histórico de demandas para o cliente C.1 após a retirada de um dado

Tabela 14 - Valores encontrados para $\Delta \mathrm{R}$ para o cliente C.1 após a retirada de um dado 
Tabela 15 - Cálculo dos desvios em relação à média para os valores do histórico de demandas para o cliente C.2

Tabela 16 - Valores encontrados para $\Delta R$ no histórico de demanda para o cliente C.2

Tabela 17 - Histórico das demandas medidas (em kW) dos clientes amostrados pertencentes ao ramo de atividade "Fabricação de produtos farmacêuticos e veterinários" sem a informação do mês de outubro de 2010

Tabela 18 - Cálculo dos desvios em relação à média para os valores do histórico de demandas do cliente C.2 após a retirada de um dado

Tabela 19 - Valores encontrados para $\Delta R$ para o cliente C.2 após a retirada de um dado

Tabela 20 - Histórico de consumo (em kWh) dos clientes amostrados pertencentes ao ramo de atividade "Fabricação de produtos farmacêuticos e veterinários"

Tabela 21 - Cálculo dos desvios em relação à média para os valores do histórico de consumo do cliente C.1

Tabela 22 - Valores encontrados para $\Delta \mathrm{R}$ no histórico de consumo para o cliente $\mathrm{C} .1$

Tabela 23 - Histórico de consumo (em kWh) dos clientes amostrados pertencentes ao ramo de atividade "Fabricação de produtos farmacêuticos e veterinários" sem a informação do mês maio de 2010

Tabela 24 - Cálculo dos desvios em relação à média para os valores do histórico de consumo do cliente C.1 após a retirada de um dado

Tabela 25 - Valores encontrados para $\Delta \mathrm{R}$ para o cliente C.1 após a retirada de um dado

Tabela 26 - Cálculo dos desvios em relação à média para os valores do histórico de consumo do cliente C.2

Tabela 27 - Valores encontrados para $\Delta \mathrm{R}$ no histórico de consumo para o cliente C.2 
Tabela 28 - Valores da demanda máxima ajustados após aplicação do critério de Chauvenet 69

Tabela 29 - Fator de demanda final por cliente 69

Tabela 30 - Fator de carga final por cliente $\quad 70$

Tabela 31 - FC e FD típico para o ramo de atividade

"Fabricação de produtos farmacêuticos e veterinários" 70

Tabela 32 - FC e FD estimados para as demais classes

de consumo do grupo A 71

Tabela 33 - Tabela de Chauvenet 103 


\section{Lista de Quadros}

Quadro 1: Localidades pertencentes a cada área geográfica da ELEKTRO

Quadro 2: Distância Euclideana (DE) entre cada área e a ELEKTRO como um todo

Quadro 3: Grupos de similaridade entre as áreas 


\section{Lista de Abreviaturas}

\begin{tabular}{|c|c|}
\hline ANEEL & Agência Nacional de Energia Elétrica \\
\hline AT & Alta tensão \\
\hline BEN & Balanço Energético Nacional \\
\hline BT & Baixa tensão \\
\hline CCEE & Câmara de Comercialização de Energia Elétrica \\
\hline EPE & Empresa de Pesquisa Energética \\
\hline FC & Fator de carga \\
\hline FD & Fator de demanda \\
\hline INMETRO & $\begin{array}{l}\text { Instituto Nacional de Metrologia, Normalização e } \\
\text { Qualidade Industrial }\end{array}$ \\
\hline MAE & Mercado Atacadista de Energia Elétrica \\
\hline ONS & Operador Nacional do Sistema \\
\hline P\&D & Pesquisa e Desenvolvimento \\
\hline $\mathrm{PPH}$ & Pesquisa de posses e hábitos de consumo \\
\hline PROCEL & $\begin{array}{l}\text { Programa Nacional de Conservação de Energia } \\
\text { Elétrica }\end{array}$ \\
\hline RESEB & $\begin{array}{l}\text { Programa de Reestruturação do Setor Elétrico } \\
\text { Brasileiro }\end{array}$ \\
\hline SINPHA & $\begin{array}{l}\text { Sistema de Informação de Posses de } \\
\text { Eletrodomésticos e Hábitos de Consumo }\end{array}$ \\
\hline
\end{tabular}

